\title{
How to be an expert in practically anything using heuristics, Bloom's taxonomy, Dreyfus model, and building rubrics for mastery: case of epidemiology and mountain bike riding
}

\author{
Arindam Basu ${ }^{1}$ \\ 1 University of Canterbury \\ Funding: The author(s) received no specific funding for this work. \\ Potential competing interests: The author(s) declared that no potential competing interests exist.
}

\section{Abstract}

In this review of how novices can develop mastery, I will summarise the key research from educational studies to the pathway of mastery. According to the five stage theory of development of mastery by Stuart and Hubert Dreyfus, everyone begin as novices. Novices in their journey to develop expertise require heuristics and rules that are context free to develop skills. With more experience and with deliberate practice with graduated challenges and feedbacks to correct errors, the novice then reaches a stage where the novice then contextualises the heuristics and rules. This is the stage where the novice then starts developing "tacit" knowledge that will enable the novice to start contextualising the problems that the novice can start to solve. In the third stage of developing mastery, the novice becomes a competent practitioner where the novice starts building an emotional attachments as they gain more experiences with a range of situations and therefore develop repertoire of skills and positions. However, at this stage, since the competent practitioner has now developed a vast range of skills and knowledge about problem solving, understanding which technique or which strategy to adopt in what situations become challenging. This stage is emotionally exhausting for the competent practitioner but the competent practitioner is now emotionally connected to the knowledge process or solving problems. Thus, if a problem is solved, the competent practitoner is elated and if the problem is not solved or the competent practitioner fails, they are emotionally upset. With more practice and development and gain of skills, experiences, the competent practitioner enters the stage of expertise. In this stage, they still analyse and use heuristics and calculations to solve problems, with even stronger emotional attachment. Finally, in stage five, when the expert becomes a master, a sense of fluidity develops, where the master is aware of, but does not need to explicitly calculate or use heuristics to solve problems, instead a sense of creativity and flow develops in the master.

Patricia Benner corroborated these concepts in the context of nursing and medical students and training of doctors. Here, the nurses and health providers undergo these five stages as they continue to develop mastery. Students start as novice nurses where they use rote learning and heuristics to solve problems. Then, as they gain more experiences in real wards and patient care, they learn how to contextualise the learned concepts in real world patient care. Over time, they develop rich repertoire of experiences and become competent practitioners to solve most day to day problems. Finally in the stage of mastery and expertise, they do not need to use the heuristics and rules anymore. 
These five-stage development of mastery can be mapped on to the concepts of cognitive development of mastery in learning that Benjamin Bloom proposed in 1956 working with school students and then he further developed as taxonomy of learning using a six-stage model. This is referred to as Bloom's taxonomy of learning mastery where the most basic stage is "remember" where the learning objective is recalling information. This is really a low level but basic activity where the students gain knowledge by rote learning but not necessarily be able to contextualise it to their learned circumstances. In the second stage of development of mastery, once the students have demonstrated the mastery of rote learning, they show that they "understand" the core concepts. In the third stage, they are able to apply the learned concepts to new and unseen or situations that they had not previously experienced. In the fourth stage, they are able to analyse novel situations and can solve problems. In the final stage, they can create new knowledge. These stages of learning mastery that Bloom proposed map on to the five-stage model of mastery development that Dreyfus proposed and Benner validated.

The question then, is, what is the mechanism by which this mastery is gained? What can a teacher do to develop expertise and mastery in a student? Bloom's concept was that it should be based on initially providing lessons, then testing whether the student has learned the concepts, and if there are broken mental models that need to be addressed by correcting errors and providing new challenges. Each time the teacher presents a new, and slightly more difficult challenge to the student; the student attempts to solve it and gains valuable experience doing so. As each stage is reached, the teacher then progresses the student to the next stage of performance. In this way, the teacher traverses the five stage of mastery by creating a mastery rubric.

We put these concepts in the context of how we can teach public health and evidence appraisal or critical thinking for students using this mastery rubric and graduated challenges. At the lowest stage, students are provided with lessons that involve rote learning and recall based assignments and formative assignments. In subsequent stages, we provide the students with more graduated challenges that they attempt to solve as they move up the ladder of expertise. Finally, the students are asked to develop a research proposal or comprehensively critique a body of knowledge.

\section{Novices, competent practitioners, and experts}

If we were to divide the world into a giant matrix of two by two, based on actual content expertise of people and what people professed they knew, we could construct a 2X2 matrix as follows (Table 1):

$<!--$ td $\{$ border: 1 px solid \#ccc;\}br \{mso-data-placement:same-cell;\}-->

Content Expertise

Self-rated expertise Do Not Know Know

Do Not Know Novices Experts

Know Competent Practitioners Masters

Such a table would bring about a conceptual category of four groupf of people, based on the intersection of their knowledge content and how they viewed the world with respect to their knowledge. Here, we use knowledge as a shorthand for their cognitive processes or content as to how much they would know about a certain topic and/or how much expertise would they posses as of "doing" or "accomplishing" a task related to that topic. In our case, we will use the 
examples of public health and say a task such as mountain bike riding. Knowledge of epidemiology can be referred to as cognitive processes where the student would need to be able to read data and patterns, obtain information from the community and arrive at activities that will save the lives of people. Mountain biking, on the other hand, is a hands-on activity where the student would need to learn sufficient details about a mountain bike and handling a mountain bike; besides, the student would need sufficient expertise in handling a mountain bike for different terrains. Based on actual expertise and self-professed expertise as shown in Table 1, we can arrive at the following classification:

- Novices DO NOT KNOW what they do not know

- Competent practitioners KNOW what they do not know

- Experts DO NOT KNOW what they know, and

- Masters KNOW what they KNOW but they have fluidity

In following this classification, we assume that whether they are novices or masters, the following hold true:

1. First of all, novices and those who are masters possess similar or comparable "quanta" of facts. For example, in case of epidemiology, the novice would normally be able to define terms and vocabulary units such as prevalence, incidence, study designs, causality in health and health care, epidemic curves, and other quanta of knowledge that are essential to the practice of everyday lives of an epidemiologist. Similarly, a novice and an expert mountain biker would be equally likely to define and describe terms such as derailleur, brakes, tube repair, terrains, and understand waypoints in maps, downhill, and other terms that are integral to the knowledge content elements of the field of mountain biking.

2. Second, while we believe using the above classification scheme defines these four classes of people based on the two axes of knowledge and self-professed knowledge (or consideration as to the "actual knowledge" and perceived knowledge), this classification is by no means static. Stewart and Hubert Dreyfus (2xxx) for example have stated that this is how expertise is built from novices to experts and masters over time. This is an evolutionary process. People start out as novices and move to become experts through a time dependent process where once one moves from one stage to the next there is no going back to the previous stage (Figure 2). So, rather than fixed statuses, these indicate progressive "stages" in which mastery is gained at each stage

3. Third, as novices "move" from being novices to gain more experiences and with practice, we see that this is a linear process that anyone, in any domain can use to gain mastery. Therefore, it is independent of age and other circumstances as long as a stepwise process of mastery can be developed.

4. Finally, everyone starts their lives as "novices" when it comes to acquiring domain expertise and no one is "born" as experts or that the process is one of "nurture" rather than "nature"; while some people may seem to be "natural" at some things, it is conceivable that they have enough experiences and trials and errors that they have devoted to gaining that expertise, and therefore, expertise is possible to be gained at any stage, wherever the stage one begins.

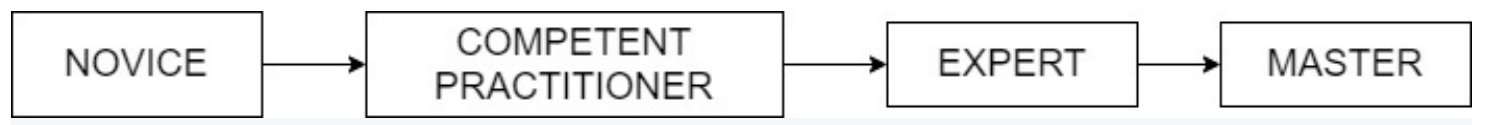

Figure 1. The direction to mastery from being a novice 
Figure 2 provides a snapshot of a simplified conceptual diagram that suggests that mastery is a continuum from being a novice through the stage of competent practitioner and expert. This should happen over time. Stuart Dreyfus (2004) has described a five stage model starting with a novice, through advanced beginner, competent practitioner, proficient in the skills and knowledge through to becoming an expert ${ }^{[1]}$

In each stage of that continuum, people in the stage are marked by some characteristics that Dreyfus provided (see Figure 3 taken from the Dreyfus' research paper):

Table 1. Five Stages of Skill Acquisition

\begin{tabular}{|c|c|c|c|c|}
\hline Skill Level & Components & Perspective & Decision & Commitment \\
\hline 1. Novice & Context free & None & Analytic & Detached \\
\hline 2. Advanced beginner & Context free and situational & None & Analytic & Detached \\
\hline 3. Competent & Context free and situational & Chosen & Analytic & $\begin{array}{l}\text { Detached understanding and deciding; } \\
\text { involved outcome }\end{array}$ \\
\hline 4. Proficient & Context free and situational & Experienced & Analytic & $\begin{array}{l}\text { Involved understanding; detached } \\
\text { deciding }\end{array}$ \\
\hline 5. Expert & Context free and situational & Experienced & Intuitive & Involved \\
\hline
\end{tabular}

Note: Components: This refers to the elements of the situation that the learner is able to perceive. These can be context free and pertaining to general aspects of the skill or situational, which only relate to the specific situation that the learner is meeting. Perspective: As the learner begins to be able to recognize almost innumerable components, he or she must choose which one to focus on. He or she is then taking a perspective. Decision: The learner is making a decision on how to act in the situation he or she is in. This can be based on analytic reasoning or an intuitive decision based on experience and holistic discrimination of the particular situation. Commitment: This describes the degree to which the learner is immersed in the learning situation when it comes to understanding, deciding, and the outcome of the situation-action pairing.

Figure 2. Skill level descriptions from Dreyfus' paper

Dreyfus identified five stages in the evolution of an expertise from a novice and identified how context-free information that a novice starts with using heuristics and detailed guides then gets transformed as with repeated practice, gaining of experiences and feedbacks where the advanced beginner then starts adding their life experiences in the mix and contextualise the knowledge. The competent practitioner stage begins when the advanced beginner develops a repertoire of rich experiences and at that point gets bewildered as to which ones to use in which context but all the time the richness of experience gets reflected in the collection of the repertoire. Once the competent practitioner starts deliberately "selecting" specific solutions to tailor to the specific situation, they move to the stage of experts but expertise marks the beginning of the phase when the expert gets emotionally involved in the solution they propose for problem solving. This is a major evolutionary leap from the competent practitioner stage to the expertise as here, creativity or experimentation to bring in new knowledge begins. Finally, when the expert transcends the experiences such that as they start solving problems they enter a state of flow (or optimal experience as time drops away ${ }^{[2]}$.

Does this five-stage model, starting with a novice who is dependent on heuristics for decision making, and then continual progression with progressively rich experience, and feedback, development of emotional intelligence and involvement to the stage of expertise work? Patricia Benner used this model for the training of nurses and reported how expert nurse clinicians, graduate students, and nurses some years in practice approach the same patients or situations. Benner has adopted the principles from Dreyfus model and made them explicit where the novices rely on heuristics and rules. The advanced beginners rely on aspects of the specific situations they encounter with their experiences. Experiences here indicate a transformation of preconceived ideas, not passage of time. The competent practitioners build on the aspects of 
experience and use guidelines to direct their actions and choose specific approaches from their repertoire, but they also adopt deliberate planning. The proficient practitioner uses more holistic approaches to specific problems and uses maxims or general principles to approach problems, while the expert is marked by intuition ${ }^{[3]}$. Tractenberg have incorporated this progressive graduated model to develop mastery rubric in a range of disciplines ${ }^{[4]}$. The Carpentries have used Dreyfus methods, combining this with Bloom's taxonomy for their training of instructors and academics/non-academics who need to work with software code, data, and literature for software carpentry, data carpentry, and library carpentry [5] The Dreyfus method is around for several decades, and additionally been validated and used in different contexts. The method has provided directions for how to develop mastery and stewardship in a range of situations such as clinical care, nursing, evidence based health, piloting aircrafts, developing software coding skills, data analysis, handling computations, and playing chess among others. However, a detailed step by step method of integrating Dreyfus method with the other extant methods such as mastery learning that Benjamin Bloom used in the sixties and on which his taxonomy of learning objectives are based on is not enunciated. Hence in this paper, I will describe some of these issues as to how to put together a plan of developing mastery in almost any domain of expertise. In the process, we will traverse the role of mental models and how mental models of novices differ from experts, and how, upon acknowledging novice-hood, we can influence mental models of individuals and specific cohorts using rubrics, assessments, and feedback processes so that anyone can design a learning system for oneself and others to ramp up to the journey of becoming an "expert" through competent practitioners from a state of novice-hood. As we start with, an understanding of the mental models and differences between novices, competent practitioners and experts/masters in their fundamental models is in order.

\section{Mental models make the difference}

We start with the assumption (see item 1 above) that novices and masters possess the same quanta of objective facts. For example, a beginning public health student and a master would work from the same or very similar vocabulary and glossary or knowledge base about populations, prevalence, incidence, causal inference or study designs. Yet what explains the apparent helplessness of a beginner epidemiologist when faced with a daunting task of investigating an epidemic and apparently effortless modelling and data analyses and guidance from a master? Or for example, a beginner mountain biker on a downhill trail will have the same quanta of knowledge about what to do or how to wield a mountain bike but the outcomes when faced with similar trails will be likely to be vastly different between a seasoned and veteran mountain biker and a new person. The clue is in the complexity of mental models of each of these individuals who are beginners and experts/masters.

A mental model can be thought of as internal representations of the reality we live in based on what we know and how we relate across different objects we know ${ }^{[6]}$. We can think of the mental models as equivalent to a network diagram (see below). The network diagram has nodes and edges. In our representation of the mental model of an individual or a group (say novices as opposed to experts), the nodes would be the facts and truths and theories that are present in any discipline. For example, for the field of epidemiology, this would mean everything we know about population, disease distribution, and how to measure diseases and determinants of diseases. For the domain of mountain biking this would indicate everything we know about how to handle mountain bikes, gears, brakes, maintenance, and so on. The edges in this network diagram are more complex. These indicate how we relate each nodes. Novices have very sparse network 
with facts that are often learned in isolation and free of context. This is also the representation of a novice's mind in Dreyfus' model (see Figure 3).

Hence for the challenge for anyone who embarks on a journey to start as a novice and end up as an expert is to progressively make the connections more dense and grow new connections. How do we progressively build connections through our experiences? The term "experience" here denotes reframing or alteration of the preconceptions that are updated with new information. How do we influence our own or other people's mental models and alter their experiential contents?

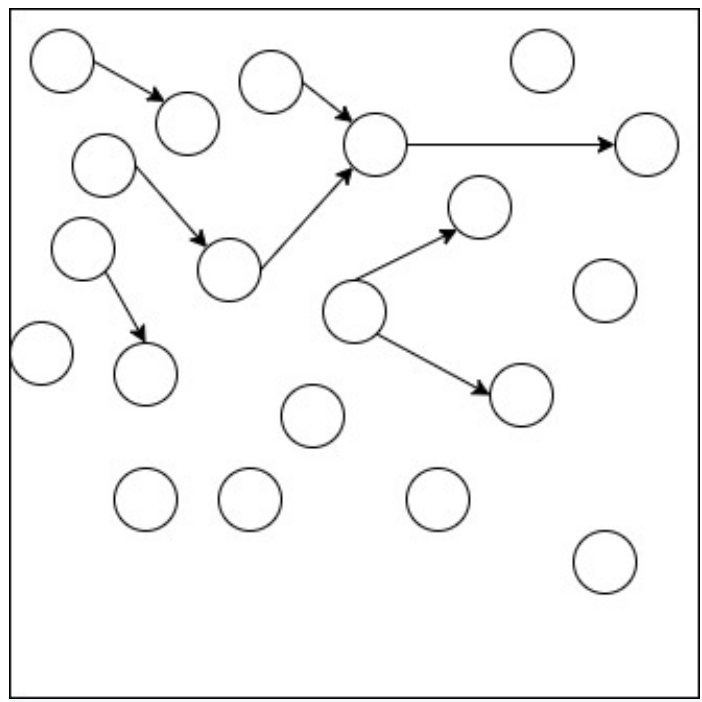

Figure 3. Mental model of a novice, circles = nodes of knowledge and arrow $=$ connection between the nodes

One way to influence our own thinking is to engage in repeated experiences, each one building on the other, and with slight variation. Each subsequent experience is slightly more complex and with feedback to guide the next steps and stages. So, deliberate practice is not the same as repeated practice with no feedback as to what our responses and errors have been ${ }^{[7]}$. Benjamin Bloom (1956) observed among school children that even though teachers taught the topics in the same way or with minimal variation, the students' performance and learning differed significantly. Bloom ascribed these differences to the repeated testing of the students, and how teachers provided feedback to correct errorsl; then once the lower domains of understanding were covered, the students moved to more cognitively challenging tasks. This laid the foundation of mastery learning ${ }^{[8]}$. In 1973 , Bloom designed a way to capture learning outcomes for students where he laid out the learning outcomes or what is expected for demonstration of learning to happen in classroom as actions along a tier of six attributes. These attributes are arranged in the form of a diagram where the tasks and attributes that are cognitively least demanding are at the base of the pyramid and tasks and actions that are cognitively challenging are placed in the top of the pyramid (Figure 4). The structure is Bloom's taxonomy of learning outcomes ${ }^{[9]}$ 


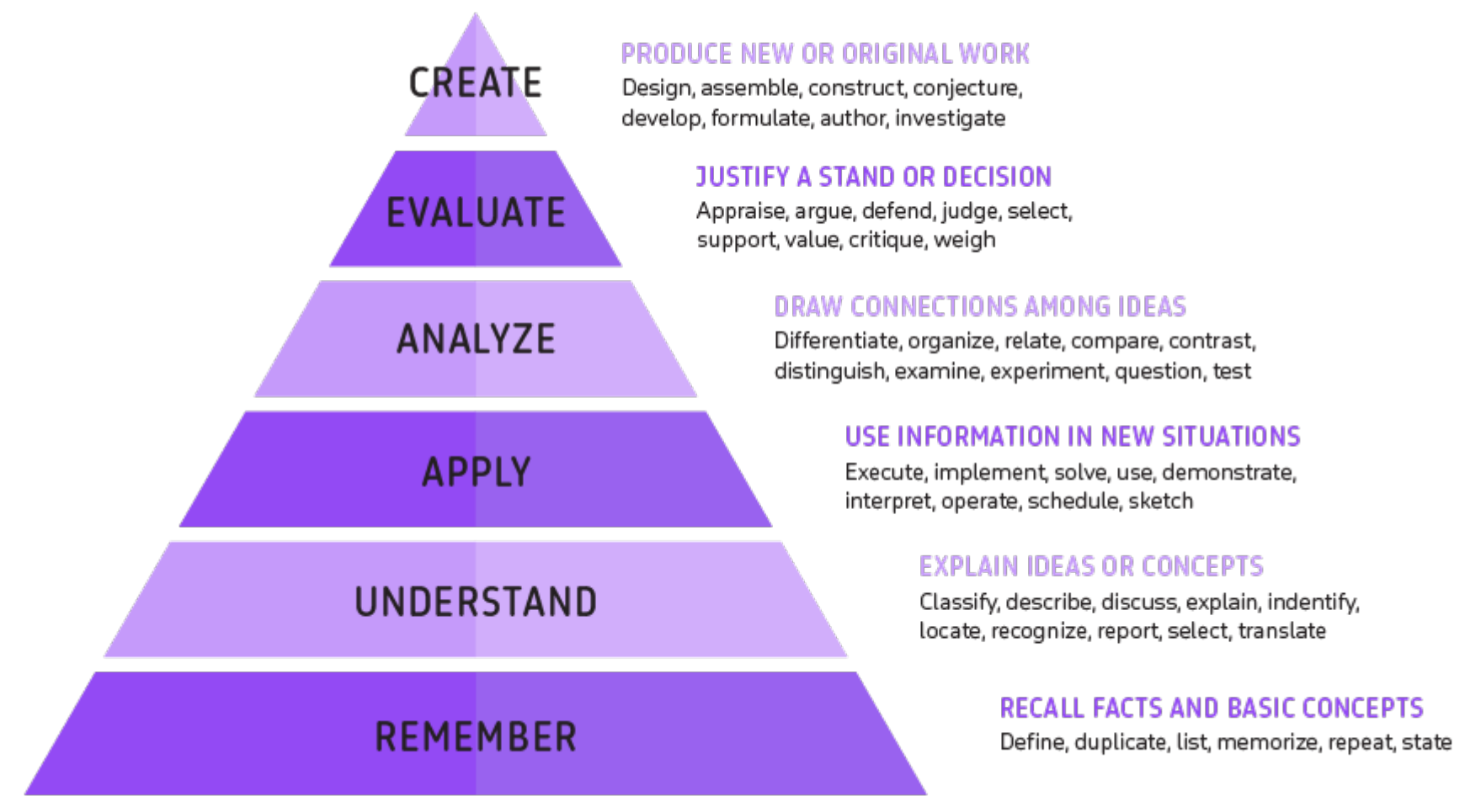

Figure 4. Bloom's taxonomy: note the pyramid shaped

So, we see here a pattern is developing as we guide either ourselves or our students from a state of novice-hood to a state of expert performer (or road to mastery). These are punctuated by five stages of acquisition of skills and marked with repeated enrichment of experiences, graduated challenges, feedback, move to the next stage after accomplishment of a set of outcomes in a preceding stage, and the cycle of learning and applying continues till the student reaches a stage where intuition starts developing. The process starts with heuristics to guide actions that are decontextualised, then the student learns two things in the process:

First, in the initial stages the heuristics and learning points are rotely memorised and instructions and directions given that are context-free, but soon, with experience and enrichment with practice, the student learns to see where the learning is situated within the deliberate practice or their life circumstances. From abstract notions of context-free heuristics, the student starts building aspects of the learning based on concrete experiences and continues to develop a repertoire of experiences where the learning topics are applied. This works with learning epidemiology, chess playing, playing a musical instrument (such as strumming guitar on chords to playing a piece of music), and something as concrete as mountain bike riding.

The second, and perhaps the more moot to this concept is that, as the student builds up a strong repertoire of "aspects" or concrete experiences and situations where specific skills must be applied, the student initially is bewildered as to what is possible but over time, learns to be focused and be selective as to which skills and which situations to prioritise. This rank ordering, this selective prioritising of the challenges and application of the learned skills to specific situations is what builds them towards mastery. As they emerge as masters, they will now move from a domain of analysis to one where they first start building maxims (or meta-approaches to solve problems) and then disband that altogether or the analyses go in the subliminal domains, and instead the student gains intuition, so all efforts are 'effortless' to an outsider. This stage also has 
some perils, as the expert develops some kind of awareness gap as to where and how the expert arrived at a solution from a problem framing. These intuitive bits make problem solving and creation effortless.

So, how would that path of progression look like in case of a novice to expert epidemiologist and a novice to an expert mountain biker? The novice epidemiologist would need a lot of heuristics to understand the nature of a problem. The formulae to calculate prevalence, estimation of sample size, guidance on designing an investigation, deliberation to choose a particular study design. These are time consuming and cognitively challenging tasks. On the other hand, to an expert this would be fluid, and the expert would be able to present the same situation in a fluid representation so that depending on the context, the expert might be able to throw the rules to the wind and yet design a new investigation or use a model to answer a critical question of public health importance using epidemiological principles. A competent practitioner on the other hand, would be able to conduct an investigation and run it effectively. Likewise, a rookie or novice mountain biker might be able to negotiate a terrain with some stopping and starting, "remembering" to loosen up as they encounter a fallen branch of a tree, and drops, but to an expert biker these would be "automatic" integrated actions on their saddles.

So, now we have covered some of these aspects where we know what moves people or their characteristics, it is time to summarise and integrate how we can put it in the context of mastery rubric and Bloom's taxonomy. The following figure shows the transitions (Figure 5)

$<!--t d$ \{border: 1px solid \#ccc;\}br \{mso-data-placement:same-cell;\}-->

\begin{tabular}{|c|c|c|c|c|}
\hline & $\begin{array}{l}\text { Novices } \\
\text { and } \\
\text { advanced } \\
\text { beginners }\end{array}$ & Competent Practitioners & Expert & Master \\
\hline Novices & -- & $\begin{array}{l}\text { Heuristics TO Context sensitivity } \\
\text { and (2) Developing Guidelines, } \\
\text { (3) select skills suited to context } \\
\text { to solve problems }\end{array}$ & Jump does not happen & Jump does not happen \\
\hline $\begin{array}{l}\text { Competent } \\
\text { Practitioners }\end{array}$ & $\begin{array}{l}\text { Do not } \\
\text { revert } \\
\text { back to } \\
\text { novices }\end{array}$ & -- & $\begin{array}{l}\text { Richer experiences and reliance on maxims as } \\
\text { opposed to guidelines, and further narrowing and } \\
\text { refinement of the selection of knowledge, skills, and } \\
\text { abilities to address problems }\end{array}$ & Jump does not happen \\
\hline Experts & $\begin{array}{l}\text { Do not } \\
\text { revert } \\
\text { back to } \\
\text { novices }\end{array}$ & do not revert & - & $\begin{array}{l}\text { Intuition and a state of flow develops, } \\
\text { analyses go to subliminal, develops a } \\
\text { state of expert or master blind spot or } \\
\text { awareness gap }\end{array}$ \\
\hline
\end{tabular}

Now that we have covered all of these various concepts of what moves a novice to a competent practitioner (deliberate practice, graduated challenges, feedback, reinforcement of growth mindset, learning goals, objectives, and outcomesfocus, and mastery rubric), let's put these in place.

\section{Sequential formative assessments to guide the path to mastery: step by step}

Step 1: Acknowledge that you are a novice or identify the novices or identify the stage of the learner and the mindset.

This step is the first step in the process. If you are designing a course for yourself or a course for someone else or a group or a class, this is a good time to sketch out who you are. Are you a budding epidemiologist who wants to solve a problem? 
Are you a new mountain bike rider? Are you someone coming back to a field later in life? There is no such thing as old age in learning. Anyone can learn anything any time. At this stage, check a couple of things:

- Make sure that the learner acknowledges the limitation of knowledge and does not suffer from what is referred to as Dunning-Kruger effect ${ }^{[10]}$. In this syndrome, someone who is not good at something would nevertheless claim that the person is good at that.

- If a student (that could be yourself) have moved past the novice hood, then check that at the stage of competent practitioner the student is not suffering from what is often referred to as impostor syndrome. This is opposite of DunningKruger effect where the performer or the student believes that they are not fit for the job. This usually happens in the context of skill acquisition where the learner's repertoire is large compared to the situations that the person has faced and one often wonders which skills to select at what point in solving problems ${ }^{[11]}$.

- This is also the stage where it is important to foster a growth as opposed to a fixed mindset. Diagnose if the student has a fixed mindset. A fixed mindset, as Carol Dweck describes is a mindset where the student refuses to learn something new or believes that there are innate and natural talents of people to learn new stuff ${ }^{[12]}$

Step 2: Design the learning outcomes appropriate for this stage.

This is important for you to understand as to where one stage (for example novices) end and say a competent practitioner starts emerging. The touch point for a competent practitioner is understanding the context sensitivity and moving out of the context-free heuristics. The competent practitioner also understands and appreciates the emotional content of the decision that person makes, and relies on guidelines that they create as opposed to the maxims or general principles. The experts on the other hand have worked out on the maxims that would guide their decisions, and the master has transcended any heuristics or analyses and instead developed intuitive knowledge or state of flow.

Also, at this stage, design the learning outcomes from simple to the more complex. This will set up the mode of delivery of your lessons. For the novice, if you want to focus only on cognitive and low level recall/remember mode, use books, movies, one-way lectures, slide decks. This is also useful for setting the heuristics. As people move up the expertise ladder, adjust your teaching to show personal practices and demonstrations and case studies. A particularly useful technique in these setting is use of participatory demonstration of the domain you want to develop. The Carpentries have used what is referred to as participatory live coding where the instructor stands up and writes codes on their computer, the codes are displayed on the projected display in the classroom and students in the workshops follow suit. Nederbragt lists ten useful tips of using this in the context of Carpentries style teaching and you can adopt these in your own teaching ${ }^{[13]}$. Use Bloom's taxonomy to design the outcomes so that easier outcomes are earlier in the lessons or training period and more challenging or cognitively demanding parts are kept for later.

Step 3: Set up your formative assessments, challenges, and feedback patterns

Once you know of your learning outcomes that will drive your learner (often yourself) to gain mastery, match your formative assessment to exactly tap into the learning outcomes and identify checkpoints where you will deliver your formative assessments. Formative assessments refer to those assessments where you identify the broken mental models of your learners and provide feedback on what they have missed or what errors they have committed. This will depend on the context in which you are setting up the training programme. In general, while teaching a classroom, you can set up a number of strategies: 
- A faded example where you can provide a fully worked out example of a solution to a problem first and then remove the "training wheels" one by one.

- Construct multiple choice questions and develop the multiple choice questions in ways where you can identify common errors in thinking or misinterpretation of the heuristics and rules you set up. Test them to make sure they understand the rules properly before moving to the next stage in the cognitive ladder of Bloom's taxonomy of outcomes

- Provide a solution first and then jumble up the solution space, so the learners will need to recreate the solution in correct order. This is generally useful when you want to teach heuristics to your students. In computer science, this is referred to as Parson's problems

- Provide them problems and ask them to solve the problem using a problem based learning framework but make sure that you have maximally provided instructions first. People at the stage of novices will like heuristics to solve problem. However, problem based learning as a teaching strategy could be useful when you are tapping either higher domains in taxonomy or when you are working with competent practitioners as you want to move them to the stage of experts [14].

Step 4: Provide them feedback to boost their outputs

This is critical as in deliberate practice where you provide either for yourself or your learners increasingly complex and real life challenges. These challenges are only as good as the feedback that accompanies them. Without feedback, a challenge loses its meaning, but equally, the nature of feedback matters. The feedback is best not directed at the person level (such as "You are great at it!"). The reason you'd need to avoid this sort of feedback is that, such feedback reinforce a fixed mindset. Instead, focus on the improvement or effort based feedback ${ }^{[15]}$.

Step 5: Set up the knowledge, skills, and ability on a mastery rubric matrix In this stage, as you have identified the learning outcome checkpoints, provided the students lessons, challenges, and feedback, and as learning keeps happening on the road to mastery it is important to chart the path to know where to call the boundaries. This is where a mastery rubric becomes important for your summative assessment of how the learning journey has been. You can create a matrix depending on the specific knowledge, skills, and abilities that you'd like to see developing in the students based on each of the stages you want to develop. For example, in the following, I have provided an example of how a mastery rubric (very simplified may look like for an epidemiology programme). Different types of learning outcomes will come out of the mastery rubric that you will set up depending on the context where this is applicable. Tractenberg (2019) has provided a comprehensive overview and guidance on how to construct mastery rubric for a range of situations (see https://osf.io/preprints/socarxiv/qd2ae/ for details).

\section{Putting it together}

In conclusion, it is fair to state that anyone in any state and stage of life can develop significant mastery in almost any subject or area of expertise, the opportunities are endless. One can develop mastery or create a suite a package for oneself or for others. It is a tool for lifelong learning.

The basic elements of developing mastery or expertise is based on the work by Dreyfus and Benjamin Bloom in different ways. Dreyfus model of expertise development is based on a student either self-identified as novice and presented initially with context-free heuristics to gain basic knowledge, and then provided with graduated challenges and feedback based on more concrete experiences. Over time, as the student gains mastery, they realise that not all aspects of the problem space 
are same, and learn to prioritise. Bloom's work provide the details of how the teaching points, challenges, and feedback are provided.

While much has been written on development of expertise and acquisiton of skills in a range of domains, the idea is that, it is possible for this to be applied everywhere. However, the extent to which is it is possible has remained under investigated. Randomised trials and other experimental study designs and meta-analyses may uncover other, nuanced, approaches that will add value to the various ways one can move from a state of being a novice to one becoming an expert.

\section{References}

1. 'Stuart E. Dreyfus. (2004). The Five-Stage Model of Adult Skill Acquisition. Bulletin of Science, Technology \& Society, vol. 24 (3), 177-181. doi:10.1177/0270467604264992.

2. 'Mihaly Csikszentmihalyi. (2018). Flow: the Psychology of Optimal Experience by Mihaly Csikszentmihalyi.

3. 'Patricia Benner. (2004). Using the Dreyfus Model of Skill Acquisition to Describe and Interpret Skill Acquisition and Clinical Judgment in Nursing Practice and Education. Bulletin of Science, Technology \& Society, vol. 24 (3), 188-199. doi:10.1177/0270467604265061.

4. ^Rochelle E. Tractenberg, Matthew M. Gushta, Jeffrey M. Weinfeld. (2016). The Mastery Rubric for Evidence-Based Medicine: Institutional Validation via Multidimensional Scaling. Teaching and Learning in Medicine, vol. 28 (2), 152165. doi:10.1080/10401334.2016.1146599.

5. ^François Michonneau and Erin Becker. The Carpentries curriculum development handbook.

6. ^Natalie A. Jones, Helen Ross, Timothy Lynam, Pascal Perez, Anne Leitch. (2011). Mental Models: An Interdisciplinary Synthesis of Theory and Methods. E\&S, vol. 16 (1). doi:10.5751/es-03802-160146.

7. ^ K. Anders Ericsson. (2008). Deliberate Practice and Acquisition of Expert Performance: A General Overview. doi:10.1111/j.1553-2712.2008.00227.x.

8. 'Thomas R. Guskey. (2005). Formative Classroom Assessment and Benjamin S. Bloom.

9. ^Benjamin Samuel Bloom, David R. Krathwohl, Bertram B. Masia. (1968). Taxonomy of Educational Objectives.

10. ^(2016). David Dunning Defines Dunning-Kruger Effect. doi:10.4135/9781473960022.

11. ^Yaniv Hozez. (2020). Impostor Syndrome. doi:10.31234/osf.io/qakwz.

12. 'Carol Dweck. (2017). What having a growth mindset means. Harvard Business Review.

13. ^Alexander Nederbragt, Rayna Michelle Harris, Alison Presmanes Hill, Greg Wilson. (2020). Ten quick tips for teaching with participatory live coding. PLoS Comput Biol, vol. 16 (9), e1008090. doi:10.1371/journal.pcbi.1008090.

14. ^Heather Purichia. (2014). Problem-Based Learning: An Inquiry Approach. Interdisciplinary Journal of Problem-Based Learning, vol. 9 (1). doi:10.7771/1541-5015.1522. 
15. 'Caroline F. Timmers, Jannie Braber-van den Broek, Stéphanie M. van den Berg. (2013). Motivational beliefs, student effort, and feedback behaviour in computer-based formative assessment. Computers \& Education, vol. 60 (1), 25-31. doi:10.1016/j.compedu.2012.07.007. 